\title{
THE EFFECTS OF INTRAVENOUSLY INFUSED VASODILATORS ON THE RENAL PLASMA FLOW AND RENAL TUBULES
}

\author{
YOON-YOUNG KIM $^{11}$ \\ Department of Pharmacology, Chung-ang University College of Medicine, \\ Seoul, Korea
}

(Received for publication July 29, 1976, introduced by Dr. M. Shingu)

Nitroglycerin increased the renal plasma flow and altered electrolyte excretion. Perhexiline, i. v, caused an increase in sodium, chloride and osmolar clearance without the changes in the renal plasma flow. An elevation of the tubular sodium rejection fraction probably contributed to the increased solute clearance.

Isoproterenol caused retention of sodium, chloride potassium, and water without changing the renal plasma flow or the glomerular filtration rate. The tubular rejection fraction of sodium was decreased, indicating that isoproterenol was directly increasing the tubular reabsorption. The renal changes induced by isoproterenol were not altered by pretreatment with perhexiline.

\section{INTRODUCTION}

Drugs which dilate the coronary arteries also affect other vascular beds. Since coronary vasodilators are prescribed for long periods of time, this effect on renal function and vasculature is an important consideration. We have reported previously on the unexpected effects of hexobendine on renal function (Cho et al., 1973). Hexobendine is a portent coronary vasodilator whose bio- . chemical mechanism of action is by inactivation of adenosine deaminase and, hence, increases the circulatory adenosine level. Hexobendine, thus, is pharmacologically similar to dipyridamole (Persantin).
In this study we selected two proto types of coronary vasodilators: nitroglycerin and perhexiline $\mathrm{HCl}$. The pharmacological properties of perhexiline have been reported before (Cho et al., 1970 ; Matsuo et al., 1970). Briefly, it possess similar, as well as dissimilar, pharmacological properties to that of nitroglycerin. Like nitroglycerin, perhexiline is a coronary vasodilator and its anti-anginal efficacy is currently being studied by others. It also has a mild bronchodilatory action, but perhaps much more so than nitroglycerin. Like nitroglycerin, while the systemic blood pressures were being minimally lowered, the heart rate was not increased but, rather, bradycardia was

1) Present Appointment : Assistant Professor, Department of Pharmacology, Chung-ang University College of Medicine, Seoul, Korea. 
observed (Cho et al., 1970). This latter aspect of perhexiline is probably due to its direct cardiac membrane action on the sinoatrial node and the atrial membrane, somewhat similar to that of quinidine (Matsuo et al., 1970).

\section{METHODS AND MATERIALS}

A group of mongel dogs, weighing 18 to $20 \mathrm{~kg}$ each were anesthetized with morphine (20 $\mathrm{mg} / \mathrm{kg}$, subcutaneously) and chlora-abdominal midline incision and the cannulac were positioned approximately $1 / 2$ inch below the uroteropelvic junction. The femoral vein and artery were cannulated and the arterial cannula was connected to a Stathum Transducer with a 3-way stopcock for recording blood pressure with a Beckman dynograph. Arterial blood samples were obtained through the 3-way stopcock. Solutions containing creatinine $(1.8 \mathrm{mg} / \mathrm{ml})$ and $\mathrm{p}$-aminohippurate $(0.5$ $\mathrm{mg} / \mathrm{ml}$ ) in normal saline were infused at the rate of $5 \mathrm{ml} / \mathrm{min}$ through the venous system by means of a dualsyringe constant-flow infusion pump. One to 2 hours were allowed for equilibration and then collections of $10 \mathrm{~min}$ urined samples from each kidney were taken. Blood samples, drawn every 20 min, were heparinized, centrifuged and the plasma immediately removed. At least three control urine samples were collected before test agents were given.

In 10 dogs, a bolus of nitroglycerin with doses ranging between $0.05,0.1$ and $0.2 \mathrm{mg} / \mathrm{kg}$., was intravenously infused through the femoral vein. Another 10 dogs received an infusion of $0.075,0.150$ and $0.300 \mathrm{mg} / \mathrm{kg}$ of perhexiline $\mathrm{HCl}$ dissolved in warm saline. In another $18 \mathrm{dog}_{\mathrm{s}}$ the additive renal effects of perhexiline and nitroglycerin were studied. Nitroglycerin, $0.05 \mathrm{mg} / \mathrm{kg}$. dissolved in 5 to $10 \mathrm{ml}$ of saline, was given intravenously to each dog. After three $10 \mathrm{~min}$ urine collections, perhexiline $(0.075 \mathrm{mg} / \mathrm{kg}$ dissolved in saline) was infused and the renal effects were studied for another $30 \mathrm{~min}$. The possibility of beta-adrenergic blocking activity of perhexiline was also studied. Isoproterenol was infused for $20 \mathrm{~min}$ at the rate of $0.1 \mathrm{ug} / \mathrm{kg} / \mathrm{min}$. Urine samples were collected every $10 \mathrm{~min}$ and blood was drawn every $20 \mathrm{~min}$.

A Buchler-Cotlove Chloridometer was used to determine plasma and urine chloride concentrations. The sodium and potassium concentrations in both plasma and urine were determined using the internal lithium standard of Model 143 Instrumentation Laboratory Flame Photometer. A modification of the Bonsnes and Taussky method (1945) was used to determine plasma and urine creatinine and PHA determinations were calculated using the technique of Bratton and Marshall (1939). Plasma and urine osmolalities were recorded by an Advanced Instruments osmometer.

\section{RESULTS}

The effects of systemically infused nitroglycerin and perhexiline, studied in two different groups of dogs, were tabulated in Table 1. Nitroglycerin caused transient hypotension occurring immediately after infusion of nitroglycerin. Perhexiline caused transient hypotension of only 3 per cent of the control, 5 minutes following infusion. The hypotension induced by perhexiline (only $3 \%$, which may not be a biologically significant change) and nitroglycerin (20 to $36 \%$ change in systolic and diastolic blood pressures, which may not be a significant change) lasted briefly. The systemic blood pressures returned to the control values within 5 minutes. 
TABLE 1

Femoral arterial blood pressures before and after intravenous infusion of Nitroglycerin (GTM) and Perhexiline (Pexid) (Mean \pm S.E.M.)

\begin{tabular}{|c|c|c|c|c|c|c|}
\hline \multirow{2}{*}{$\begin{array}{c}\text { Blood } \\
\text { pressure }\end{array}$} & \multirow{2}{*}{\multicolumn{2}{|c|}{ Control }} & \multicolumn{4}{|c|}{ Min after i. v. drugs } \\
\hline & & & Immediately & 5 & 10 & 30 \\
\hline Systolic & $\begin{array}{c}\mathrm{GTN}(0.2 \\
\mathrm{mmHg} \\
\%\end{array}$ & $\begin{array}{c}\mathrm{g} / \mathrm{kg} \text { i.v. }) \\
181.0 \pm 8.72 \\
-\end{array}$ & $\left|\begin{array}{r}145.0 \pm 9.75^{*} \\
-19.9 \pm 4.34^{*}\end{array}\right|$ & $\left|\begin{array}{l}174.0 \pm 7.65 \\
-3.8 \pm 0.50 *\end{array}\right|$ & $\begin{array}{l}176.0 \pm 6.96 \\
-2.6 \pm 1.12\end{array}$ & $\begin{array}{l}179.0 \pm 9.80 \\
-1.2 \pm 0.75\end{array}$ \\
\hline Diastolic & $\underset{\%}{\mathrm{mmHg}}$ & $\begin{array}{c}124.0 \pm 9.54 \\
-\end{array}$ & $\left|\begin{array}{r}79.0 \pm 14.70^{*} \\
-35.6 \pm 9.31^{*}\end{array}\right|$ & $\begin{array}{l}125.0 \pm 8.80 \\
+1.0 \pm 1.48\end{array}$ & $\begin{array}{l}124.0 \pm 8.28 \\
+0.3 \pm 2.23\end{array}$ & $\begin{array}{r}126.6 \pm 9.60 \\
+2.2 \pm 1.69\end{array}$ \\
\hline Mean & $\underset{\%}{\mathrm{mmHg}}$ & $\begin{array}{r}143.0 \pm 9.10 \\
-29.7 \pm 6.85\end{array}$ & $\left|\begin{array}{r}101.0 \pm 12.82^{*} \\
-12.0 \pm 0.84^{*}\end{array}\right|$ & $\begin{array}{l}141.2 \pm 8.42 \\
-0.9 \pm 1.22\end{array}$ & $\begin{array}{r}141.4 \pm 7.83 \\
+0.5 \pm 1.11\end{array}$ & $143.8 \pm 9.60$ \\
\hline Systolic & $\begin{array}{c}\text { Perhexiline } \\
\mathrm{mmHg} \\
\%\end{array}$ & $\begin{array}{c}0.3 \mathrm{mg} / \mathrm{kg} \mathrm{i} . \mathrm{v} .) \\
183.0 \pm 11.14 \\
-\end{array}$ & $\begin{array}{c}183.0 \pm 11.14 \\
0\end{array}$ & $\begin{array}{l}177.0 \pm 9.43 \\
-3.1 \pm 0.91 *\end{array}$ & $\begin{array}{l}179.0 \pm 10.30 \\
-2.1 \pm 1.56\end{array}$ & $\begin{array}{l}180.0 \pm 11.73 \\
-1.7 \pm 1.11\end{array}$ \\
\hline Diastolic & $\underset{\%}{\mathrm{mmHg}}$ & $\begin{array}{c}127.4 \pm 9.50 \\
-\end{array}$ & $\begin{array}{r}127.0 \pm 9.03 \\
-0.2 \pm 1.38\end{array}$ & $\begin{array}{l}123.0 \pm 8.16 \\
-3.2 \pm 1.36^{*}\end{array}$ & $\begin{array}{l}123.0 \pm 9.60 \\
-2.2 \pm 2.48\end{array}$ & $\begin{array}{l}121.0 \pm 11.00 \\
-2.3 \pm 2.01\end{array}$ \\
\hline Mean & $\underset{\%}{\mathrm{mmHg}}$ & $\begin{array}{c}145.8 \pm 9.85 \\
-\end{array}$ & $\begin{array}{r}145.6 \pm 9.53 \\
-0.1 \pm 0.89\end{array}$ & $\begin{array}{l}141.0 \pm 8.57 \\
-3.1 \pm 0.94^{*}\end{array}$ & $\begin{array}{l}141.6 \pm 9.03 \\
-2.7 \pm 2.06\end{array}$ & $\begin{array}{r}140.8 \pm 10.98 \\
-2.0 \pm 1.54\end{array}$ \\
\hline
\end{tabular}

* = P less than 0.05 or less.

$\%=$ The per cent change in blood pressure measured immediately following i. v. infusion of the drugs 5, 10 and 30 min after the drug infusion has been calculated against the control values.

The values obtained in the renal clearance studies are tabulated in Tables 2,3 and 4 . The control variables or renal plasma flow $(\mathrm{ml} / \mathrm{min})$, glomerular filtration rate $(\mathrm{ml} / \mathrm{min})$, osmolar clearance $(\mathrm{ml} / \mathrm{min})$, urine volume $(\mathrm{ml} / \mathrm{min}$ $\left.\times 10^{-2}\right)$, Sodium $(\mathrm{mEq} / \mathrm{min})$, potassium ( $\mathrm{mEq} / \mathrm{min})$, chloride $(\mathrm{mEq} / \mathrm{min})$, the tubular rejection fraction of sodium, and filtration fraction are compatible with published data (Bencasath et al., 1971 ; Williams and Pearson, 1970).

Nitroglycerin, $0.05 \mathrm{mg} / \mathrm{kg}$, caused no changes except a minimal kaliuretic action (Table 2). Marked natriuretic, kaliuretic and chloriuretic effects, as well as increases in the osmolar clearance rates, were observed in dogs receiving $0.2 \mathrm{mg} / \mathrm{kg}$ of nitroglycerin, i. v.
(Table 2). At this dose level the renal plasma flow rate was also increased. Perhexiline, at a dose level of 0.075 $\mathrm{mg} / \mathrm{kg}$, caused no change in renal function. However, perhexiline, at a dose level of $0.300 \mathrm{mg} / \mathrm{kg}$, caused a significant increase in urinary sodium and chloride excretion rates, as well as increases in osmolar clearance. Unlike nitroglycerin, RPF was not altered at all dose levels (Table 3 ).

The results measured after $0.05 \mathrm{mg} /$ $\mathrm{kg}$ of nitroglycerin showed an increase in renal plasma flow $(+19.2 \%)$ and glomerular filtration rate $(+15.7 \%)$. All other variables were not significantly altered.

When $0.075 \mathrm{mg} / \mathrm{kg}$ of perhexiline $\mathrm{HCl}$ was added, there were significant in- 
TABLE 2

The renal clearance studies after Nitroglycerin, $I . V$.

\begin{tabular}{|c|c|c|c|c|c|c|c|}
\hline $\begin{array}{l}\text { Nitro- } \\
\text { glycerin }\end{array}$ & $\mathrm{RPF}$ & GFR & $\mathrm{C}_{\text {osm }}$ & 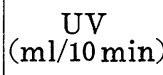 & & & \\
\hline Control & $95.0 \pm 8.27$ & $40.3 \pm 10.01$ & $3.70 \pm 0.287$ & $42.5 \pm 8.05$ & $428.0 \pm 50.02$ & $88.3 \pm 3.84$ & $397.5 \pm 28.14$ \\
\hline \multirow{2}{*}{$0.05 \mathrm{mg} / \mathrm{kg}$} & $104.8 \pm 6.86$ & $45.0 \pm 8.04$ & $3.84 \pm 0.320$ & $41.0 \pm 5.61$ & $469.2 \pm 46.76$ & $98.2 \pm 1.80$ & $447.8 \pm 24.67$ \\
\hline & $(7.4 \pm 4.58)$ & $(4.3 \pm 4.58)$ & $(3.6 \pm 2.14)$ & $(-0.6 \pm 6.49)$ & $(5.2 \pm 2.31)$ & $(10.0 \pm 4.64)$ & $(10.4 \pm 2.98)$ \\
\hline \multirow{2}{*}{$0.1 \mathrm{mg} / \mathrm{kg}$} & $110.7 \pm 5.52$ & $50.0 \pm 5.65$ & $4.76 \pm 0.292$ & $42.8 \pm 1.54$ & $592.6 \pm 47.83$ & $125.4 \pm 4.86$ & $\begin{array}{r}\mathrm{xx} \\
565.6 \pm 32.17\end{array}$ \\
\hline & $(11.0 \pm 1.35)$ & $(10.7 \pm 3.58)$ & $(21.7 \pm 2.14)$ & $(21.2 \pm 9.83)$ & $(28.6 \pm 3.52)^{\mathrm{X}}$ & $\begin{array}{r}\mathrm{xxx} \\
(38.2 \pm 3.44)\end{array}$ & $(36.7 \pm 3.76)$ \\
\hline \multirow{2}{*}{$0.2 \mathrm{mg} / \mathrm{kg}$} & $104.0 \pm 7.07$ & $45.8 \pm 7.19$ & $5.18 \pm 0.51$ & $47.3 \pm 1.52$ & $635.3 \pm 78.16$ & $\begin{array}{r}\mathrm{xxx} \\
134.0 \pm 8.12\end{array}$ & $\begin{array}{r}\mathrm{xxx} \\
636.8 \pm 51.47\end{array}$ \\
\hline & $(9.4 \pm 1.11)$ & $(18.4 \pm 6$. & $(42.6 \pm 4.77)$ & $(21.7 \pm 4.82)$ & $(46.4 \pm 5.93)$ & $(50.8 \pm 4.66)$ & $(59.4 \pm 6.09)$ \\
\hline
\end{tabular}

TABLE 3

The renal clearance studies after Perhexiline $H C l, I . V$.

\begin{tabular}{|c|c|c|c|c|c|c|c|}
\hline \multirow{2}{*}{ Perhexiline } & \multicolumn{4}{|c|}{$\mathrm{ml} / \mathrm{min}$} & \multicolumn{3}{|c|}{$\mathrm{uEq} / \mathrm{min}$} \\
\hline & $\mathrm{RPF}$ & GFR & $\begin{array}{l}\mathrm{C} \\
\text { osm }\end{array}$ & {$\left[\begin{array}{c}\mathrm{UV} \\
\mathrm{ml} / 10 \mathrm{~min}\end{array}\right.$} & $\mathrm{Ua}_{\mathrm{Na}}^{\mathrm{V}}$ & $\mathrm{U}_{\mathrm{K}}^{\mathrm{V}}$ & $\mathrm{Ul}_{\mathrm{Cl}}^{\mathrm{V}}$ \\
\hline Cor & 103.0 & & $3.93 \pm 0.192$ & $62.3 \pm 4.19$ & $465.3 \pm 47.27$ & $106.0 \pm 9.65$ & $484.8 \pm 51.44$ \\
\hline 0.07 & $\begin{array}{l}103.0 \pm 3.81 \\
(-0.2 \pm 2.68)\end{array}$ & $\begin{array}{c}46.3 \pm 5.66 \\
(19.2 \pm 3.56)\end{array}$ & $\begin{array}{c}4.31 \pm 0.187 \\
(9.5 \pm 1.60)\end{array}$ & $\begin{array}{l}63.8 \pm 3.07 \\
(2.1 \pm 2.65)\end{array}$ & $\left|\begin{array}{l}536.3 \pm 41.59 \\
(16.0 \pm 3.45)\end{array}\right|$ & $\mid \begin{array}{r}110.5 \pm 98.4 \\
(4.1 \pm 0.94)\end{array}$ & $\begin{array}{l}544.8 \pm 46.49 \\
(12.8 \pm 3.26)\end{array}$ \\
\hline $\mathrm{g} / \mathrm{kg}$ & $\begin{array}{c}105.0 \pm 5.76 \\
(1.3 \pm 3.20)\end{array}$ & $\begin{array}{c}45.7 \pm 5.19 \\
(14.6 \pm 5.97)\end{array}$ & $\begin{array}{r}\mathrm{xxx} \\
4.88 \pm 0.097 \\
\mathrm{x} \\
(24.6 \pm 3.85)\end{array}$ & $\begin{array}{r}65.0 \pm 1.71 \\
(4.9 \pm 3.57)\end{array}$ & $\mid \begin{array}{r}x \\
(31.3 \pm 4.5 \pm 31.90\end{array}$ & $\begin{array}{l}116.2 \pm 6.16 \\
(10.0 \pm 1.81)\end{array}$ & $\left\{\begin{array}{r}x \\
(31.1 \pm 6.85)\end{array}\right.$ \\
\hline $0.300 \mathrm{mg} / \mathrm{kg}$ & $\begin{array}{c}97.8 \pm 4.44 \\
(-3.7 \pm 2.65)\end{array}$ & $\begin{array}{c}40.9 \pm 2.38 \\
(11.1 \pm 4.50)\end{array}$ & $\begin{array}{r}\mathrm{xxx} \\
5.52 \pm 0.209 \\
\mathrm{xxx} \\
(37.7 \pm 3.02) \\
\end{array}$ & $\begin{array}{r}64.0 \pm 1.67 \\
\times \times x \\
(0 \pm 2.18)\end{array}$ & $\begin{array}{r}\mathrm{xx} \\
759.8 \pm 48.46 \\
(56.7 \pm 4.44)\end{array}$ & $\begin{array}{l}106.5 \pm 4.31 \\
(-2.4 \pm 2.49)\end{array}$ & $\begin{array}{r}\mathrm{Xxx} \\
737.9 \pm 42.96 \\
\mathrm{xxx} \\
(46.3 \pm 3.81)\end{array}$ \\
\hline
\end{tabular}

creases in renal plasma flow $(+20.9 \%)$, glomerular filtration rate $(+17.8 \%)$, osmolar clearance $(+20.7 \%)$, sodium clearance $(+34.4 \%)$, and chloride clearance $(+28.5 \%)$.

The infusion of isoproterenol at a rate of $0.1 \mathrm{ug} / \mathrm{kg} / \mathrm{min}$ for $21 \mathrm{~min}$ caused a marked reduction in osmolar clearance $(74.1 \%)$, urine volume $(46.3 \%)$, sodium clearance $(63.0 \%)$, potassium clearance $(54.5 \%)$, chloride clearance
$(+59.2 \%)$, and tubular rejection fraction of sodium (69.2\%). Perhexiline failed to block the renal effects of isoproterenol.

\section{DISCUSSION}

A pharmacologically active dose of nitroglycerin $(0.2 \mathrm{mg} / \mathrm{kg})$ caused an increase in the renal plasma flow and 
TABLE 4

The renal clearance studies after Nitroglycerin $(0.05 \mathrm{mg} / \mathrm{kg})$ Perhexiline $(0.075 \mathrm{mg} / \mathrm{kg})$ and Isoproterenol $(0.1 \mathrm{mg} / \mathrm{kg} / \mathrm{min})(N=18)(\%=$ changes to the control) and absolute values (Mean \pm S.E.M.)

\begin{tabular}{|c|c|c|c|c|c|c|c|c|c|}
\hline \multirow[b]{2}{*}{ Drugs } & \multicolumn{4}{|c|}{$\mathrm{ml} / \mathrm{min}$} & \multicolumn{3}{|c|}{$\mathrm{uEq} / \mathrm{min}$} & \multirow{2}{*}{$\begin{array}{l}\text { TRF } \\
\quad \mathrm{Na}\end{array}$} & \multirow{2}{*}{ F F } \\
\hline & $\mathrm{RPF}$ & GMR & $\mathrm{C}_{\text {osm }}$ & $\begin{array}{c}(\mathrm{Vol}) \\
-2+ \\
\times 10\end{array}$ & $\begin{array}{c}\mathrm{U}_{\mathrm{Na}}^{\mathrm{V}} \\
\end{array}$ & $\mathrm{U}_{\mathrm{K}}^{\mathrm{V}}$ & $\mathrm{U}_{\mathrm{Cl}}^{\mathrm{V}}$ & & \\
\hline \multirow[b]{2}{*}{ Control } & $(100)$ & $(100)$ & (100) & (100) & (100) & (100) & $(100)$ & (100) & (100) \\
\hline & $\begin{array}{l}107.6 \\
\pm 13.35\end{array}$ & $\begin{array}{l}53.3 \\
\pm 5.19\end{array}$ & $\begin{array}{l}3.99 \\
\pm 0.461\end{array}$ & $\begin{array}{l}34.6 \\
\pm 3.07\end{array}$ & $\begin{array}{l}480.3 \\
\pm 71.39\end{array}$ & $\begin{array}{l}85.5 \\
\pm 9.52\end{array}$ & $\begin{array}{l}470.1 \\
\pm 67.63\end{array}$ & $\begin{array}{l}9.77 \\
\pm 2.011\end{array}$ & $\begin{array}{l}0.51 \\
\pm 0.026\end{array}$ \\
\hline \multirow{2}{*}{ GTN } & $\mid \begin{array}{l}*(119.2) \\
( \pm 6.70)\end{array}$ & $\begin{array}{l}*(115.7) \\
( \pm 5.17)\end{array}$ & $\begin{array}{l}(110.1) \\
( \pm 7.67)\end{array}$ & $\begin{array}{l}(96.5) \\
( \pm 3.99)\end{array}$ & $\begin{array}{l}(111.5) \\
( \pm 10.74)\end{array}$ & $\begin{array}{l}(108.0) \\
( \pm 4.66)\end{array}$ & $\begin{array}{l}(110.0) \\
( \pm 8.48)\end{array}$ & \begin{tabular}{|l|}
$(99.6)$ \\
$( \pm 0.04)$
\end{tabular} & $\begin{array}{l}(97.8) \\
( \pm 3.30)\end{array}$ \\
\hline & $\begin{array}{l}123.3 \\
\pm 15.48\end{array}$ & $\begin{array}{l}57.7 \\
\pm 4.81\end{array}$ & $\begin{array}{l}4.26 \\
\pm 0.456\end{array}$ & $\begin{array}{l}33.6 \\
\pm 3.73\end{array}$ & $\begin{array}{l}408.1 \\
\pm 65.22\end{array}$ & $\begin{array}{l}90.2 \\
\pm 10.36\end{array}$ & $\begin{array}{l}500.2 \\
\pm 66.58\end{array}$ & $\begin{array}{l}90.02 \\
\pm 1.237\end{array}$ & $\begin{array}{l}0.49 \\
\pm 0.025\end{array}$ \\
\hline \multirow{2}{*}{ Pexid } & $\mid \begin{array}{l}*(120.9) \\
( \pm 9.47)\end{array}$ & $\begin{array}{l}*(117.8) \\
( \pm 6.06)\end{array}$ & $\begin{array}{l}(120.7) \\
( \pm 9.96)\end{array}$ & $\begin{array}{l}(106.1) \\
( \pm 5.76)\end{array}$ & $\left(\begin{array}{l}(134.4) \\
( \pm 16.60)\end{array}\right.$ & $\begin{array}{l}(98.5) \\
( \pm 6.99)\end{array}$ & $\begin{array}{l}*(128.5) \\
( \pm 12.73)\end{array}$ & $\begin{array}{l}(114.8) \\
( \pm 12.70)\end{array}$ & $\begin{array}{l}(99.0) \\
( \pm 3.60)\end{array}$ \\
\hline & $\begin{array}{l}126.5 \\
\pm 14.31\end{array}$ & $\begin{array}{l}61.6 \\
\pm 5.04\end{array}$ & $\begin{array}{l}4.64 \\
\pm 0.465\end{array}$ & $\begin{array}{l}36.7 \\
\pm 3.97\end{array}$ & $\begin{array}{l}592.6 \\
\pm 65.92\end{array}$ & $\begin{array}{l}80.7 \\
\pm 8.60\end{array}$ & $\begin{array}{l}471.5 \\
\pm 62.19\end{array}$ & $\begin{array}{l}9.56 \\
\pm 1.071\end{array}$ & $\begin{array}{l}0.50 \\
\pm 0.022\end{array}$ \\
\hline \multirow{2}{*}{ Iso } & $\begin{array}{l}(89.5) \\
( \pm 10.98)\end{array}$ & $\begin{array}{l}*(88.8) \\
( \pm 12.24)\end{array}$ & $\begin{array}{l}(\ddot{H}(74.1) \\
\quad( \pm 13.79)\end{array}$ & $\begin{array}{l}*(46.3) \\
( \pm 10.55)\end{array}$ & $\begin{array}{l}*(63.0) \\
( \pm 17.31)\end{array}$ & $\begin{array}{l}*(54.5) \\
( \pm 13.19)\end{array}$ & $\begin{array}{l}*(59.2) \\
( \pm 14.73)\end{array}$ & $\begin{array}{l}*(69.2) \\
\quad( \pm 12.87)\end{array}$ & $\begin{array}{l}(97.1) \\
( \pm 4.22)\end{array}$ \\
\hline & $\begin{array}{l}95.4 \\
\pm 16.54\end{array}$ & $\begin{array}{l}45.3 \\
\pm 6.41\end{array}$ & $\begin{array}{l}2.32 \\
\pm 0.477\end{array}$ & $\begin{array}{l}16.1 \\
\pm 4.20\end{array}$ & $\begin{array}{l}254.3 \\
\pm 64.46\end{array}$ & $\begin{array}{l}45.9 \\
\pm 6.91\end{array}$ & $\begin{array}{l}240.3 \\
\pm 58.61\end{array}$ & $\begin{array}{l}5.53 \\
\pm 1.009\end{array}$ & $\begin{array}{l}0.49 \\
\pm 0.022\end{array}$ \\
\hline
\end{tabular}

$*=(\mathrm{p} 0.06$ or less $)$

$\#=(0.1 \mathrm{p} 0.05)$

$\mathrm{RPI}=\mathrm{PAH}$ clearance (renal plasma flow) in $\mathrm{ml} / \mathrm{min}$;

$\mathrm{GFR}=\mathrm{g}$ lomerular filtration rate in $\mathrm{ml} / \mathrm{min}$;

$($ Vol. $)=$ brine volum in $\mathrm{ml} / \mathrm{min} \times 10^{-2} ; \quad \mathrm{U} \mathrm{Na} \mathrm{V}=$ sodium excretion $(\mathrm{uEq} / \mathrm{min})$;

$\mathrm{UK} \mathrm{V}=$ potassium excretion $(\mathrm{uEq} / \mathrm{min}$ ); $\quad \mathrm{U} \mathrm{Cl} \mathrm{V}=$ chloride excretion ( $\mathrm{uEq} / \mathrm{min}$ );

$\mathrm{C}$ osm $=$ osmolar clearance $(\mathrm{ml} / \mathrm{min}) ; \quad \mathrm{TRF} \mathrm{Na}=$ tubular rejection praction of sodium ;

$\mathrm{FF}=$ filtration fraction

electrolyte excretion rate. Tubular sodium rejection fraction was not altered.

Intravenous infusion of $0.3 \mathrm{mg} / \mathrm{kg}$ of perhexiline $\mathrm{HCl}$ did not increase renal plasma flow. There was an increase in sodium, chloride and osmolar clearance which would be attributed to an increase in renal flow. An elevation of the tubular sodium rejection fraction was not great enough to be statistically significant, but may well explain the saluretic effect of perhexiline which was not accounted for by increased renal flow. This saluretic effect of per- hexiline may explain the clinical weight loss in 69 patients receiving perhexiline for 8 weeks (Unpublished data). The weight loss has been noted by others (Hirshleifer, 1969) and is not due to anorexia (Hudak et al., 1970).

The additive renal effects of nitroglycerin $(0.05 \mathrm{mg} / \mathrm{kg})$ and perhexiline $\mathrm{HCl} \quad(0.075 \mathrm{mg} / \mathrm{kg})$ were interesting findings. Nitroglycerin caused enhancement of the perhexiline-induced renal effects at an ineffective dose level. The saluretic effects and osmolar clearance rate were increased by perhexiline at 
the dose level of $0.075 \mathrm{mg} / \mathrm{kg}$, which was an ineffective dose of perhexiline without the presence of nitroglycerin.

These additive renal effects of perhexiline to nitroglycerin and the renal action observed after nitroglycerin alone, or perhexilin alone, suggest that these two coronary vasodilators may act through two different mechanisms in the kidney. Nitroglycerin, at effective dose levels, may increase natriuresis, kaliuresis and chloriuresis by changing the renal plasma flow rate, whereas perhexiline may be a mild diuretic agent acting directly upon the renal tubules at its effective dose levels. Renal plasma flow was not altered by perhexiline.

Perhexiline possesses certain pharmacological actions which mimic betaadrenergic blocking agents. In canine heart-lung preparations $(20 \mathrm{mg} / \mathrm{kg}$ ) it reduced left ventricular work (Cho et al., 1970). Perhexiline will modify exercise - induced tachycardia in healthy volunteers (Bunde et al., 1969) and angina patients (Winsor, 1970). In isolated guinea pig atria a dose dependent increase in rate caused by histamine, acetylcholine and isoproterenol can be blocked by perhexiline (Tasi, 1969).

However, in other areas, perhexiline does not mimin beta-adrenergic blocking agents. In patients with bronchial asthma perhexiline $\mathrm{HCl}$ will cause bronchodilation. The data compiled in this study shows that perhexiline does not prevent or modify the renal effects induced by isoproterenol, a beta-stimulant.

The infusion of isoproterenol after perhexiline caused a decrease in urine volume and osmolal, sodium, potassium and chloride clearance without altering renal plasma flow rate or glomerular filtration. The infusion of $0.1 \mathrm{ug} / \mathrm{kg} /$ min in the femoeral vein over a $20 \mathrm{~min}$ period caused an increase in tubular reabsorption and, therefore, a decrease in tubular rejection fraction of sodium. However, other investigators have noted that a lower dose level infused slowly in to the renal artery will induce diuresis and natriuresis, while a higher dose at a faster rate will induce salt and water retension (Mark et al., 1969). Apparently, high doses of isoproterenol stimulate vasoconstriction in canine renal vasculature. However, in clinical studies, isoproterenol was found to increase effective renal plasma flow in patients with heart failure (Sandler et al., 1961) and pulmonary emphysema (Saltzman et al., 1961)

In conclusion, we have thus far studied three coronary vasodilators whose pharmacological mechanisms of action are different. Nitroglycerin, a prototype vasodilator of all the other nitrates and nitrities ; hexobendine (Cho et al., 1973), a prototype vasodilator of one whose action is by inactivation of adenosine deaminase and increasing coronary flow, such as dipyridamile (Persantin), prenylamine (Segantin), etc. Finally, perhexiline $\mathrm{HCl}$ may represent a totally new class of antianginal drug. Perhexiline may increase the coronary flow while it may not increase the heart rats in the face of slightly lowered systemic blood pressure (Cho et al., 1970). Also, perhexiline originally was thought to have some "sympatholytic" activities, such as inducing a slight degree of bradycardia in dogs (Cho et al., 1970) and causing a modification in tachycardia induced by exercise in volunteers (Bunde et al., 1969). In isolated tissue preparations, perhexiline demonstrates certain antagonistic effects to those agonists such as isoproterenol, histamine and acetylcholine(Unpublished data). Furthermore, perhexiline possesses a cardiac membrane effect grossly similar to that of quinidine. For the time being, this 
agent may be classified as a "coronary vasodilator" acting directly upon the ringed vascular smooth muscles.

Nitroglycerin, at a relatively higher dose level, may cause a slight degree of natriuretic action because of the increased renal plasma flow rate. Perhexiline, on the other hand, does not increase the renal plasma flow but may act directly upon the renal tubules to reject the sodium. There are additive renal effects between nitroglycerin and perhexiline compensating one another.

Hexobendine apparently causes an antidiuretic activity, acting through its direct effect on the renal tubules.

\section{ACKNOWLEDGEMENT}

I wish to thank Prof. Y. W. Cho $[$ Professor and chairman, Department of Pharmacology, Chung-ang University College of Medicine, Seoul, Korea ; and University of North Carolina School of Medicine in Chapel Hill, U.S.A. (Associate Professor of Clinical Pharmacology and Pharmacology)] for his advice and guidance throughout this study.

\section{REFERENCES}

Bunde, C. A., Crupp, I.L. and Grupp, C. (1969). Effects of the antianginal agent perhexiline maleate on exercise induced tachycardia in human volunteers. Fed. Proc., 28, 672.

Botting, R., FARMer, J.B. and Lockett, M.F. (1961). The effect of subcutaneous adrenalin and isoprenalin on the excretion of electrolytes by rats. Arch. Int. Physiol. Biochem., 69, 203.

Bratton, A.C. and Marshall, E.K., Jr. (1939). A new coupling component of sulfanilamide determination. J. Biol. Chem., 128, 537.

Bencasath, P., Szslsy, L., Demeczky, L. and TAKACS, L. (1971). Effects of chlorothiazide and furosemide on sodium and water excretion after unilateral splanchnictomy in the dog. Nephron, 8, 329.

Bonsnes, K.W. and TAussKy, H.H. (1945). On the colorimetric determination of creatinine by the Jaffe reaction. J. Liol. Chem., 158, 581.

Cho, Y.W., Belej, M. and Aviado, D. M. (1970).
Pharmacology of a new antianginal drug: Perhexiline. I . Coronary circulation and myocardial metabolism. Chest, 58, 577.

Cho, Y.W. Pearson, J.E. and Saito, S. (1973). The direct and systemic effects of hexobendine on renal function. Aroh. Int. Pharmacodyn, 201, 217.

Feinsilver, O, Cho, Y.W. and Aviado, D.M. (1970). Pharmacology of a new antianginal drug: Perhexiline. III. Bronchopulmonary system in the dog and human. Chest, 58, 448.

Hudak, W. J., Kuhn, W. L. and Lewis, R. E. (1970). Cardiovascular activity of perhexiline. J. Pharmacol. Exp. Therap., 173, 364.

Hirshleifer, I. (1969). Perhexiline maleate in the treatment of angina pectoris. Curr. Therap. Res., 11, 99.

Matsuo, S., Cho, Y.W. and Aviado, D.M. (1970). Pharmacology of a new antianginal drug: Perhexiline. II. Heart rate and transmembrane potential of cardiac tissue. Chest, $\mathbf{5 8}$, 581.

MARK, A.L., Eckstein, J.W., Abboud, F.M. and WENDLING, M. G. (1969). Renal vascular responses to osiproterenol. Am. J. Physiol. 217, 764.

Pearson, J. E. and Williams, R. L. (1968). Analysis of direct renal actions of alpha and beta adrenergic stimulation upon sodium excretion compared to acetylcholine. Brit. J. Pharmacol. Chemotherap., 33, 223. Sandler, H., Hodge, H. T., Jr. and Murdaugh, H.V. (1961). Effects of isoproterenol on cardiac output and renal function in congestive heart failure. Am. Heart J., 62, 643. Saltzman, H., Manfredi, F. and Sieker, H.O. (1961). Renal hemodynamic changes in pulmonary emphysema. J. Lab. Clin. Med., 57, 694.

TASI, T. H. (1969). The effect of perhexiline $\mathrm{HCl}$ on the pacemaker of the isolated guinea pig atria. Pharmacologist, 11, 244.

Unpublished Data, Filed with the NationalMerrell Research Laboratorie, Cincinnati, Ohio (Pexid and Antianginal Efficacy).

WINsoR, T. (1970). Clinical evaluation of perhexiline maleate. Clin. Pharmacol. Therap., 11,85 .

Williams, R. L. and Pearson, J. E. (1970). Renal effects of a vasodilator:2-(N-methylpiperidyl-(4.)) -3-amino-5-(4'-pyridyl) -pyrazole $\mathrm{HCl}$ (Ciba 31-531 Ba). J. Pharmaceut. Sci, 59, 183. 